\title{
Corrigendum: Creation of a two-dimensional electron gas at an oxide interface on silicon
}

\author{
J.W. Park, D.F. Bogorin, C. Cen, D.A. Felker, Y. Zhang, C.T. Nelson, C.W. Bark, C.M. Folkman, X.Q. Pan, \\ M.S. Rzchowski, J. Levy \& C.B. Eom
}

Nature Communications 1:94 doi: 10.1038/ncomms1096 (2010); Published 19 Oct 2010; Updated 5 Feb 2013.

The financial support for the work described in this Article was not fully acknowledged. The Acknowledgements should have read:

We thank J. Mannhart, S. Thiel and D.G. Schlom for helpful discussions. The authors gratefully acknowledge the financial support of the National Science Foundation through grants DMR-0906443 (C.B.E.), DMR-0704022 (J.L.), ARO W911NF-08-1-0317 (J.L.), DARPA Seedling (J.L.), Fine Foundation (J.L.), DMR-0907191 (X.Q.P.), DoE/BES DE-FG02-07ER46416 (X.Q.P.), Nanochip and David and Lucile Packard Fellowship (C.B.E.). One of the authors (X.Q.P.) acknowledges the support of the National Center for Electron Microscopy, Lawrence Berkeley Lab, which is supported by the US Department of Energy under Contract \# DE-AC02-05CH11231. 